\title{
Adjuvant Chemotherapy and Prognostic Factors in Stage II Colon Cancer - Izmir Oncology Group Study
}

\author{
Yuksel Kucukzeybek ${ }^{1 *}$,Ahmet Dirican ${ }^{1}$, Lutfiye Demir ${ }^{1}$, Serkan Yildirim² $^{2}$, Murat \\ Akyol $^{1}$, Yasar Yildiz ${ }^{1}$, Ibrahim Vedat Bayoglu ${ }^{1}$, Ahmet Alacacioglu ${ }^{1}$, Umut Varol ${ }^{1}$, \\ Tarik Salman' ${ }^{1}$, Ibrahim Yildiz ${ }^{1}$, Huseyin Can ${ }^{3}$, Mustafa Oktay Tarhan ${ }^{1}$
}

\begin{abstract}
Background: Although adjuvant chemotherapy is a standard treatment in stage III colon cancer, its benefit is not as clear for stage II patients. In this retrospective analysis, we aimed to evaluate the survival of patients with low-risk stage II colon cancer, the efficacy of adjuvant chemotherapy in high-risk stage II colon cancer patients, and prognostic factors in stage II disease. Materials and Methods: One hundred and seventeen patients who were diagnosed with stage II colon cancer between January 2006 and December 2011 were included in the study. Patients were stratified into two groups as being low-risk and high-risk according to risk factors for stage II disease. Adjuvant 5-fluorouracil-based chemotherapy were administered to the patients with risk factors. Results: Ninety-four patients were treated with adjuvant chemotherapy due to high risk factors and 23 were monitored without treatment. Median follow-up time was 43 months. In terms of disease free survival and overall survival, adjuvant chemotherapy did not provide a statistically significant difference. Univariate analysis demonstrated that bowel obstruction was the major risk factor for shortened disease-free survival, while bowel perforation and perineural invasion were both negative prognostic factors for overall survival. Conclusions: The recommendation of adjuvant chemotherapy for stage II colon cancer is not clear. In our study, it was found that adjuvant chemotherapy did not contribute to survival in high-risk stage II patients. Due to the fact that prognosis of stage II patients is good, many more patients will be needed for statistically significant differences in survival. Adjuvant chemotherapy containing 5 fluorouracil is being used to high-risk stage II patients although it is not a standard treatment approach.
\end{abstract}

Keywords: Colon cancer - stage II - - adjuvant therapy - prognostic factors

Asian Pac J Cancer Prev, 16 (6), 2413-2418

\section{Introduction}

Colorectal cancer is the third most frequent cause of cancer-related deaths both in women and men and the incidence 1.2 million /per year (Siegel et al., 2014; Ferlay et al., 2010). In developing countries, incidence of colorectal cancer has been reported higher than the other countries (Talaiezadeh et al., 2013). It is well known that the disease stage is the major prognostic factor for colorectal cancer. Forty percent of colon cancer patients have lymph node involvement in initial diagnosis, however $15-20 \%$ of the patients with T3-T4 invasion do not lymph node involvement (Eisenberg et al, 1982).

Surgery is the mainstay of curative intent for locoregional colorectal cancer and adjuvant chemotherapy is the standard recommended treatment for stage III colon cancer patients. However, the importance of adjuvant treatment for stage II colon cancer is controversial.
American Society of Clinical Oncology (ASCO) guidelines, do not routinely recommend adjuvant chemotherapy for stage II patients while in European Society of Medical Oncology (ESMO) guidelines, it is specified that adjuvant chemotherapy can be used for selected stage II patients (Benson et al., 2004; Labianca et al., 2013). The results of various studies demonstrated that the factors such as T4 disease, poorly differentiated histology, lymphovascular invasion, perineural invasion, bowel obstruction or perforation, surgical margin positivity, and inadequate lymph node sampling were related with poor prognosis in stage II colon cancer (Quah et al., 2008; Gill et al., 2004; Chen et al., 2000; Compton et al., 2000; Chang et al., 2007).

In this study, the efficacy and toxicity profile of adjuvant chemotherapy on high-risk stage II colon cancer patients and the survival of low-risk stage II colon cancer patients were retrospectively evaluated. 


\section{Materials and Methods}

\section{Patients}

The medical records of the patients who were diagnosed with resected colon cancer and admitted to Izmir Katip Celebi University, Ataturk Training and Research Hospital, Medical Oncology out-patient clinic between January 2006 and December 2011 were examined. Among 795 patients, the patients with stage I,III and IV disease were excluded. Subsequently the remaining 134 stage II patients were evaluated separately. Among 134 patients with stage II disease 17 of them were excluded who had lost follow-up. Remaining 117 patients were included in the study. The clinicopathologic (age, gender, the presence of bowel obstruction or perforation, tumor histologic type and grade, the number of resected and involved lymph nodes, the presence of lymphovascular invasion, perineural invasion, surgical margin positivity ) data were assessed. High-risk stage II colon cancer patients were defined as the patients who had at least one of the following risk factors: T4 invasion, presence of lymphovascular invasion, presence of perineural invasion, inadequate number of dissected lymph nodes (less than 12), presence of obstruction or perforation. Adjuvant chemotherapy was administered to these patients. Patients who did not carry any of the above risk factors were defined as low-risk group, and these patients were followed without treatment.

\section{Treatment procedure}

5 fluorouracil based chemotherapy was administered to the high-risk stage II colon cancer patients. MAYO regimen (six cycles, in each 28 day cycle) was used as adjuvant treatment procedure (bolus $425 \mathrm{mg} / \mathrm{m}^{2}$ of 5 fluorouracil on days $1-5$ and $20 \mathrm{mg} / \mathrm{m}^{2}$ of folinic acid on days 1-5 ). All patients underwent complete physical examination and toxicity assessment (including blood counts and biochemical parameters) on every pre-treatment period. Patients were evaluated for hematological and non-hematological toxicities and were graded according to the National Cancer Institute (NCI) Common Toxicity Criteria. The following treatment cycle was administered if there was no evidence of disease progression and the following criteria were provided: hemoglobin $9.0 \mathrm{~g} / \mathrm{dL}$ (after transfusion if necessary), the number of neutrophils $\geq 1,500 / \mu \mathrm{L}$, and platelets $\geq 100,000 / \mu \mathrm{L}$. After completion of adjuvant chemotherapy, all patients were monitored with routine physical examination, blood counts, biochemical parameters, and carcinoembryonic antigen every 3 months. Colonoscopy were performed every $1 \mathrm{st}$ year, $3^{\text {rd }}$ year and then fifth year. Annual thorax-abdomenpelvic CT examination was carried out for 5 years to patients who received adjuvant chemotherapy. Low-risk patients were evaluated using radiological examination on suspicion of recurrence.

\section{Statistical evaluation}

DFS (disease-free survival) was defined as the time from the initial diagnosis to the disease recurrence or the last visit which came first. OS (overall survival) was defined as the time from the initial diagnosis to the death or last visit (which came first). All statistical analyses were performed using SPSS for Windows (Statistical Package for Social Sciences, Chicago, IL). P values $<0.05$ were considered statistically significant. Survival analyses were performed by using Kaplan-Meier curves. Survival difference between two groups according to different variables were determined using the log-rank test. The effect of each significant predictor identified via univariate analysis was assessed via using cox's proportional hazards model.

\section{Results}

Median follow-up time was 43 months (10-79). $65.8 \%$ of the patients $(n=77)$ were male and median age was 65 years old (range:37-80). The majority of the patients had T3 disease $(93.2 \%, \mathrm{n}=109)$ and $80.3 \%$ of patients $(n=94)$ had well-differentiated tumours. $17.9 \%$ of the patients $(n=21)$ presented with the clinic of bowel obstruction and $3.4 \%(n=4)$ had bowel perforation at initial diagnosis. Inadequate number of lymph node dissection was performed in $28.7 \%$ of patients $(n=33) .94$ patients $(80.3 \%)$ received adjuvant chemotherapy due to being considered as high-risk and 23 low-risk patients (19.7\%) were followed up without treatment. Mean duration of chemotherapy was 4.24 (SD+/-2.31) months, median cycle of chemotherapy was 6(2-6). The patient and tumor characteristics are summarized in Table 1.

\section{Disease free survival}

In terms of evaluating all of the patients with resected stage II colon cancer diagnosis, 1, 3. 5-year disease-free survival rates were determined as $96.7 \%, 90.5 \%$ and $88.6 \%$ respectively (Figure 1). 5-year DFS rate of the patients

Table 1. Patient Demographics and Baseline Characteristics

\begin{tabular}{lc}
\hline & \% of Patients (n:117) \\
\hline Median age, years & 65 \\
Age category, years & \\
$\quad<65$ & 48.7 \\
$\quad \geq 65$ & 51.3 \\
Sex & \\
$\quad$ Male & 65.8 \\
$\quad$ Female & 34.2 \\
Depth of invasion & \\
$\quad$ T3 & 93.2 \\
T4 & 6.8 \\
Perforation present & 3.4 \\
Bowel obstruction present & 17.9 \\
Histology & \\
$\quad$ Differentiated & 80.3 \\
Poorly differentiated & 6 \\
$\quad$ Unknown & 13.7 \\
Lymphovascular invasion & 17.8 \\
Perineuronal invasion & 5.9 \\
Number of lymph nodes sampled & \\
$\quad<12$ & 28.7 \\
$\quad \geq 12$ & 71.3 \\
Adjuvant Chemoterapy & \\
$\quad$ Yes & 80.3 \\
No & 19.7 \\
\hline
\end{tabular}


who received adjuvant chemotherapy was found to be $87.5 \%$. The effects of the following prognostic factors on relapse risk were investigated by univariate analyses separately: the receipt of adjuvant chemotherapy $(\mathrm{p}=0.611$, HR: 1.70, 95\%CI:0.21-13.6) (Figure 2), the effect of age $(<65$ or $\geq 65)(\mathrm{p}=0.610$, HR: $0.98,95 \%$ CI: 0.933-1.04), gender $(\mathrm{p}=0.532$, HR: $0.656,95 \% \mathrm{CI}: 0.175-2.46)$, the presence of poor differentiated tumours $(\mathrm{p}=0.863$, HR: $1.295 \% \mathrm{CI}: 0.15-9.5)$, the presence of lymphovascular invasion ( $\mathrm{P}=0.343, \mathrm{H}: 2.02$ 95\% CI:0.50-8.09), T4 invasion $(\mathrm{p}=0.691$, HR: $1.51,95 \% \mathrm{CI}: 0.19-12.1)$, the presence of inadequate lymph node dissection $(\mathrm{p}=0.486$, HR: 1.638 , 95\% CI:0.409-6.559). No statistically significant DFS difference could be detected among above variables.

Table 2. Toxicities of the Chemotherapy

\begin{tabular}{lcc}
\hline Toxicity & Grade1-2(\%) & Grade 3-4 (\%) \\
\hline Leukopenia & 80.4 & 1.1 \\
Neutropenia & 79.9 & 7.6 \\
Thrombocytopenia & 80.4 & 1.1 \\
Diarrhea & & 8.5 \\
Nausea and vomiting & & 5.1 \\
Stomatitis & & 9.4 \\
\hline
\end{tabular}

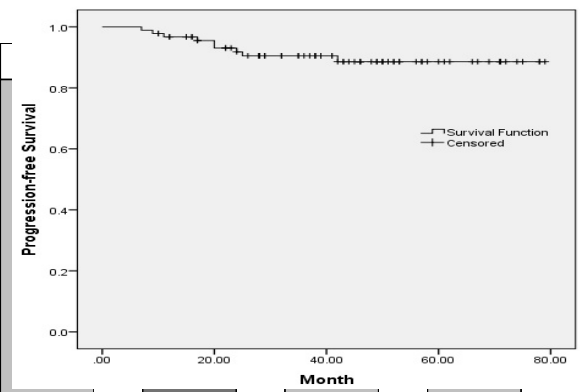

Figure 1. Disease Free Survival

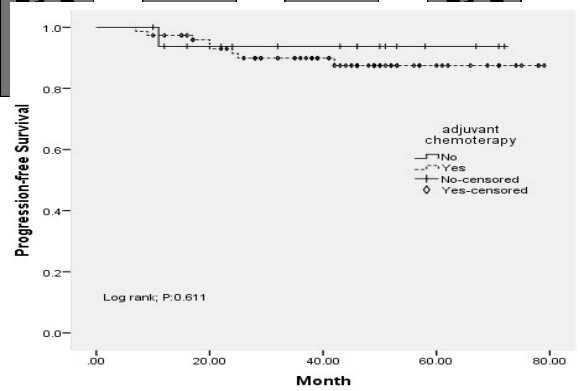

Figure 2. Disease Free Survival for Patients who did Receive and did not Receive Adjuvant Chemotherapy

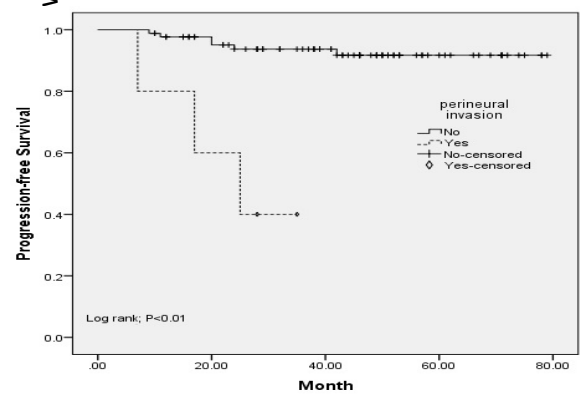

Figure 3. Disease Free Survival Analysis According to the Parameter: Perineural Invasion
DOI:http://dx.doi.org/10.7314/APJCP.2015.16.6.2413 Adjuvant Chemotherapy and Stage II Colon Cancer Prognosis However, the presence of perineural invasion ( $\mathrm{p}<0.001$, HR: 13.4 95\%CI:3.1-25) (Figure 3), bowel obstruction $(\mathrm{p}=0.035$, HR: 4.1, 95\%CI:1.10-15.3) (Figure 4), and bowel perforation ( $\mathrm{p}<0.001$, HR: 16.4, 95\%CI:3.8137.4) (Figure 5), had significantly negative impact on the prognosis of stage II colon cancer patients; these patients had significantly shorter DFS times when compared to the patients who did not have these risk factors.

\section{Overall survival}

In terms of evaluating all of the patients with resected stage II colon cancer, 1, 3. 5-year overall survival rates were identified as $98.9 \%, 94.5 \%$ and $93.1 \%$ respectively (Figure 6). 5-year OS rate of patients who received adjuvant chemotherapy was $92.8 \%$. When the effects of the traditional risk factors on death risk were investigated by univariate analysis no significant overall survival difference could be demonstrated among the following variables ; the receipt of adjuvant chemotherapy ( $\mathrm{p}=0.744$, HR:1.42, 95\%CI:0.171-11.8), age ( $\mathrm{p}=0.901$, HR:0.909, 95\% CI:0.203-4.06), gender ( $\mathrm{p}=0.072$, HR:0.221, 95\%CI:0.043-1.14), the presence of poor differentiated tumours $(\mathrm{p}=0.700$, HR:1.51, 95\%CI:0.182-12.6), inadequate lymph node dissection

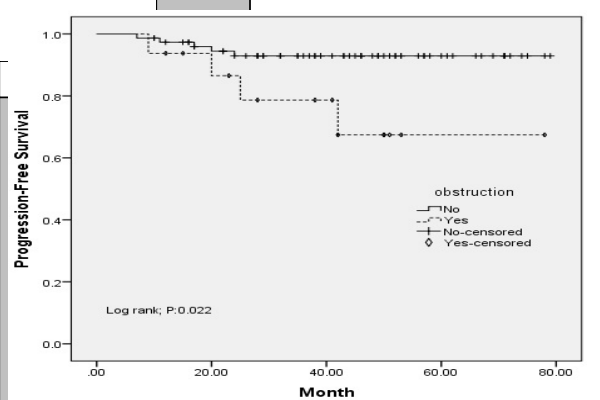

Figure 4. Disease Free Survival by the Presence of Obstruction

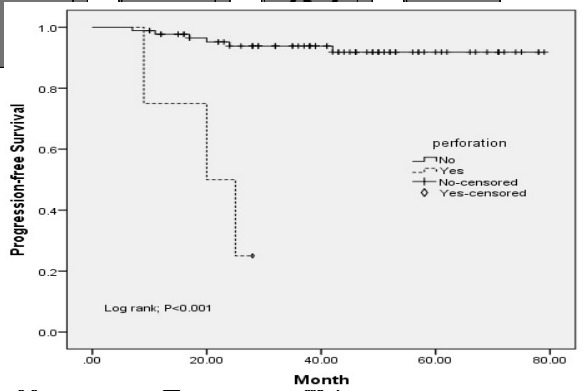

Figure 5. Disease Free Survival by the Presence of Perforation

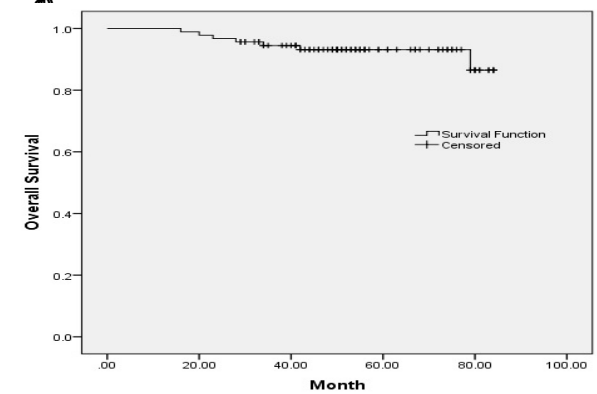

Figure 6. Overall Survival 


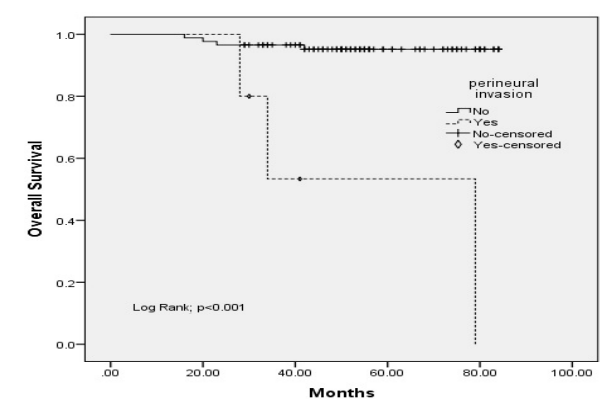

Figure 7. Overall Survival by the Presence of Perineural Invasion

( $\mathrm{P}=0.201$, HR:3.98, 95\%CI:0.478-33.25), lymphovascular invasion $(\mathrm{p}=0.078, \mathrm{HR}: 3.93,95 \% \mathrm{CI}: 0.85-18.03)$, T4 invasion ( $\mathrm{p}=0.463$, HR:2.21, 95\%CI:0.266-18.3), bowel obstruction ( $\mathrm{p}=0.459$, HR:1.858, 95\%CI:0.360-9.59). However, the patients who presented with the tumours with perineural invasion ( $\mathrm{p}<0.001, \mathrm{HR}$ : 15.4 95\%CI:3.3371.9) (Figure 7) and with the clinic of bowel perforation at initial diagnosis(p <0.002, HR: 15.59, 95\% CI:2.78-47.39) (Figure 8 ) had significantly shorter overall survival times compared to the patients without these factors.

\section{Toxicity}

Grade1-2 leukopenia and grade3-4 leukopenia was observed in $80.4 \%$ and $1.1 \%$ of the patients respectively. $73.9 \%$ of the patients had grade1-2 neutropenia, $7.6 \%$ were grade $3-4$ neutropenia, $80.4 \%$ were grade1-2 thrombocytopenia, $1.1 \%$ were grade $3-4$ thrombocytopenia. When considered non hematological toxicities, it was observed that $8.5 \%$ had grade 3-4 diarrhea, 5.1\% had grade3-4 nausea and vomiting, $9.4 \%$ had grade 3-4 stomatitis. The type and severity of toxicities observed during adjuvant chemotherapy is summarized in Table II

\section{Discussion}

5Fluorourasil-based adjuvant chemotherapy is used as standard in colon cancer diagnosed patients since 1990's (Moertel et al., 1990). Adjuvant 5Fluorourasil-based chemotherapy is recommended as the standard therapy for resected stage III colon cancer patients. However, the administration of adjuvant chemotherapy for patients with stage II colon cancer is controversial (Benson et al., 2004; Labianca et al., 2013). Adjuvant chemotherapy studies including both stage II and stage III colon cancer patients could not demonstrate a similar statistically significant survival benefit for stage II patients as for stage III patients. IMPACT B2 study, which is a pooled analysis of five studies including 1016 patients evaluated the efficacy of adjuvant bolus 5Fluorourasil combined with folinic acid in the patients with resected stage II colon cancer. 5-year disease-free survival rates were $73 \%$ and $76 \%$, and overall survival rates were $80 \%$ and $82 \%$ for adjuvant chemotherapy and control groups respectively. Thus, in terms of disease-free survival and overall survival, no significant benefit of adjuvant chemotherapy could be shown. Advanced age and the presence of poorly differentiated tumor are found to be associated with poor prognosis. When the toxicity is evaluated, it is specified that the grade 3-4 toxicities nausea and vomiting are reported in rate of $4 \%$, stomatitis is $11 \%$, diarrhea is $8 \%$, leucopenia is $2 \%$ and thrombocytopenia is reported in rate of 2\%. In QUASAR study (Quick and Simple and Reliable Study) the efficacy of bolus 5fluorourasil were evaluated among 3239 patients of whom $71 \%$ had colon cancer and among colon cancer patients $91 \%$ had stage II disease. The chemotherapy was found to be effective in preventing disease recurrence for first 2 years among whole study group $(\mathrm{p}<0.001)$, however no DFS benefit after 2 years was observed $(\mathrm{p}=0.94)$. Similar significant benefit in disease recurrence for the first two years was also observed in stage II patients $(\mathrm{p}=0.01)$. Additionally relative risk of death for stage II patients was 0.84 which was statistically significant $(\mathrm{p}=0.046)$ (Gray et al., 2007). Thus, in QUASAR study, the benefit of bolus 5fluorourasil-based chemotherapy for stage II patients was reported both in recurrence-free survival and overall survival. However, among stage II patients there were patients with rectum cancer diagnoses as well. Moreover, after the completion of the study, the survival results of stage II colon cancer were not announced separately. Therefore, the results of Quasar study are not sufficient to recommend the usage of 5fluorourasil-based adjuvant chemotherapy in all patients with stage II colon cancer. Cakar et al retrospectively evaluated the efficacy of 5 fluorouracil based chemotherapy both in low-risk and high-risk stage II colon cancer patients. No statistically significant DFS difference between the two groups was observed (Cakar et al., 2013). Trivedi et al evaluated the prognostic factors and survival of patients with stage II colon cancer retrospectively. There was no statistically significant difference between treatment group and control group. In that study, age was only exhibited to be significantly in relationship with OS (Trivedi $\mathrm{H}$ et al., 2014). A statistically significant DFS difference was detected in another study evaluating the prognostic factors and adjuvant chemotherapy in patients with high risk stage II colon cancer. In multivariate analysis, patient age $>60$ years and $\mathrm{T} 4$ tumor stage have been reported as independent prognostic factors in terms of DFS (Artac M et al., 2014). In our study, consistent with the literature, we could not establish any significant difference in DFS rates between the adjuvant chemotherapy group (that has at least one of the prognostic risk factors), and control group (lowrisk group or adjuvant chemotherapy administered patient group that does not have risk factors) $(\mathrm{p}=0.611)$. 5-year DFS and OS of the entire patient group (including low risk and high risk groups) was $88.6 \%$. and $93.6 \%$ respectively. Thus, in the chemotherapy group the administration of adjuvant chemotherapy did not provide any benefit on overall survival rates $(\mathrm{p}=0.744)$. When side effect profile of 5 fluorouracil-based adjuvant chemotherapy that was administered is evaluated, conforming with the literature data, $1.1 \%$ grade $3-4$ leucopenia, $1.1 \%$ grade 3-4 thrombocytopenia, 7.6\% grade 3-4 neutropenia, $8 \%$ grade 3-4 diarrhea, 5\% grade 3-4 nausea and vomiting, $10 \%$ grade 3-4 stomatitis was observed.

In the pooled analysis of National Surgical Adjuvant Breast and Bowel Project (NSABP) adjuvant colon cancer 
studies (C01-C05), the effect of adjuvant 5 fluorouracilbased chemotherapy in colon cancer patients were assessed. Among $42 \%$ of the patients with stage II disease, a significant benefit (for both DFS and OS) was observed in patients who received 5 fluorourasil-based chemotherapy $(\mathrm{p}<0.001)$ (Wilkinson et al., 2010). Similarly, a systematic review in which 12 randomized controlled studies were assessed, it is found that 5fluorourasil-based chemotherapy has extended both DFS and OS in stage II colon cancer significantly (Wu et al., 2012).

Despite the fact that adjuvant chemotherapy is not recommended for whole stage II colon cancer patients, it is well known that the majority of the previous adjuvant studies (comparing 5FU only with oxaliplatin based combination studies) included an important number of stage II patients. MOSAIC study, in which $40 \%$ of the patients had stage II disease investigated the benefit of adding oxaliplatin to the infusional 5fluorourasil. Initial results of this study demonstrated that survival advantage was prominent in only stage III patients, and adding oxaliplatin to 5fluorourasil- based chemotherapy did not have any effect on DFS and OS times for stage II patients (André et al., 2004). Recently reported long-term results of MOSAIC study established that T4 disease, poorly differentiated tumours, bowel obstruction/perforation, venous invasion, presence of less than 10 lymph node sampling were significant risk factors for stage II disease. Thus, the patients who had at least one of these risk factors were considered as high-risk. However, for both low-risk and high-risk stage II patients, adding oxaliplatin to the infusional 5 fluorourasil did not cause any significant difference in terms of survival. In high-risk patient group, 5-year DFS and 6-year OS rates were reported as $82.3 \%$ and $85 \%$ respectively (Tournigand et al., 2012). In C07 study, in which the efficacy of adding oxaliplatin to bolus 5fluorourasil was evaluated, approximately $30 \%$ of the patients had stage II disease. The results of this study was reported in 2007 and demonstrated a significant DFS advantage in entire patient population (Kuebler et al., 2007). Similar with the results of MOSAIC study, long term results of C07 study established that DFS benefit was observed in stage 111 patients only, however no OS or DFS advantage was observed in stage II patients. Fiveyear DFS and OS rates of stage II patients were reported as $82.1 \%$ and $89.7 \%$ respectively. It was shown that in the group to whom bolus 5 fluorourasil was administered $32.4 \%$ of grade 3-4 diarrhea, $11 \%$ of grade 3-4 nausea, $7.9 \%$ of grade 3-4 vomiting have occurred (O'Connor et al., 2011). In our study, similar DFS and OS rates were obtained with bolus 5 fluorourasil. Similar side effects were observed in patients who received chemotherapy.

Some of the reported risk factors for patients with stage II colon cancer are preoperative CEA elevation, T4 disease, poorly differentiated histology, lymphovascular invasion, perineural invasion, bowel obstruction or perforation, surgical margin positivity, inadequate lymph node sampling, and these risk factors are established to be related with poor prognosis (Quah et al., 2008; Gill et al., 2004; Chen et al., 2000; Compton et al., 2000; Chang et al., 2007; Baek JY et al., 2014). Lai et al evaluated clinical significance of lymphovascular invasion by Adjuvant Chemotherapy and Stage II Colon Cancer Prognosis D2-40 antibody and CD34 antibody staining compared with regular hematoxylin eosin staining in patients with stage II colorectal cancer and reported that death risk of patients with positive D2-40 and CD34 combination was 5 times that of double negative patients (Lai et al., 2014). However, the presence of risk factors does not predict the effectiveness of adjuvant chemotherapy. In another study, which is a pooled analysis of 7 randomized studies including 3,302 stage II and stage III colon cancer patients, nodal status, $\mathrm{T}$ stage and tumour grade were reported as prognostic factors. In another study, in which 24,847 patients with resected stage II colon cancer were evaluated, obstruction, perforation, T4 stage, poorly differentiated histology, presence of less than 12 lymph node sampling has been considered as poor prognostic factors. It is reported that adjuvant chemotherapy does not have statistically significant contribution on the OS in the stage II disease (O'Connor et al., 2011). We also evaluated the prognostic significance of these risk factors in our study population. In univariate analysis, the presence of bowel obstruction had a significant effect on development of earlier disease recurrence. Moreover, the presence of perforation and perineural invasion were both found to be statistically related with shortened DFS and OS. Nowadays studies analyzing other prognostic factors have been carried out in patients with stage II colorectal cancer. Ozdemir et al demonstrated that increasing pretreatment neutrophil lymphocyte ratio was associated with decreased overall survival in patients with operable colorectal cancer (Ozdemir et al., 2014). Meng et al evaluated whether allogeneic red blood cell transfusions showed a deleterious effect in patients with stage II colon cancer and reported that nontransfused patients has a better survival rate than patients who were transfused 1 to 3 units. In that study, 10 year mortality rate has been found as 20\% (Meng et al., 2013). Baghestani et al evaluated competing risk factors including body mass index, height, age at diagnosis, tumor site and gender in patients with colorectal cancer and concluded that age at diagnosis has statistically significant effect on survival of patients. Patients with age under 53 years have been shown to have longer survival (Baghestani et al., 2014).

In the literature there is a little evidence suggesting the efficacy of adjuvant chemotherapy in stage II colon cancer. Therefore, the factors that will predict the efficacy of adjuvant chemotherapy are being investigated. Microsatellite instability reflecting defective mismatch repair is found in around $15 \%$ of the colon cancer patients. In the study in which microsatellite instability is assessed within stage II and stage III cancers, MSI was found to be a prognostic marker, but 5fluorourasil-based chemotherapy did not provide any OS benefit in patients with high-frequency microsatellite instability. However there was a significant prolongation in OS times in the patients with low-frequency microsatellite instability or microsatellite-stable tumors (Ribic et al., 2003). In the outcome of the study in which defective mismatch repair in stage II and stage III colon cancer was assessed, the patients with stage III colon cancer that has proficient MMR did benefit from the treatment; on the other hand, in terms of presence of defective MMR, patients did not 
benefit from the treatment. In addition, it is indicated that presence of dMMR or pMMR does not predict the response to the treatment in stage II patients (Sargent et al., 2010).

In conclusion, 5fluorourasil -based adjuvant chemotherapy is not a standard strategy for stage II colon cancer. Due to the fact that prognosis of stage II patients are good, many more patients that will make statistically significant difference in survivals cannot be included to the studies. Adjuvant chemotherapy containing 5 fluorouracil is being used to high-risk stage II patients although it is not standard treatment approach. Eventhough various prognostic factors are identified in stage II disease, factor that might predict the treatment response is not defined yet.

\section{References}

André T, Boni C, Mounedji-Boudiaf L, et al (2004). Oxaliplatin, fluorouracil, and leucovorin as adjuvant treatment for colon cancer. N Engl J Med, 350, 2343-51.

Artac M, Turhal NS, Kocer M, et al (2014). Do high risk features support the use of adjuvant chemotherapy in stage II colon cancer? a turkish oncology group study. Tumori, 100, 143-8.

Baek JY, Yeo HY, Chang HJ, et al (2014). Serpin B5 is a CEA interacting biomarker for colorectal cancer. Int J Cancer, 134, 1595-604.

Baghestani AR, Daneshvar T, Pourhoseingholi MA, Asadzade H (2014). Survival of colorectal cancer patients in the presence of competing-risk. Asia Pac J Cancer Prev, 15, 6253-5.

Benson AB 3rd, Schrag D, Somerfield MR, et al (2004). American society of clinical oncology recommendations on adjuvant chemotherapy for stage II colon cancer. J Clin Oncol, 22, 3408-19.

Cakar B, Varol U, Junushova B, et al (2013). Evaluation of the efficacy of adjuvant chemotherapy in patients with high risk stage II colon cancer. JBUON, 18, 372-376.

Chang GJ, Rodriguez-Bigas MA, Skibber JM, et al (2007). Lymph node evaluation and survival after curative resection of colon cancer: systematic review. J Natl Cancer Inst, 99, 433-41.

Chen HS, Sheen-Chen SM, (2000). Obstruction and perforation in colorectal adenocarcinoma: an analysis of prognosis and current trends. Surgery, 127, 370-6.

Compton CC, Fielding LP, Burgart LJ, et al (2000). Prognostic factors in colorectal cancer. College of American Pathologists Consensus Statement 1999. Arch Pathol Lab Med, 124, 979-94.

Eisenberg B, Decosse JJ, Harford F, et al (1982). Carcinoma of the colon and rectum: the natural history reviewed in 1704 patients. Cancer, 49, 1131-4.

Ferlay J, Shin HR, Bray F, et al (2008). Estimates of worldwide burden of cancer in 2008: GLOBOCAN 2008. Int J Cancer, 127, 2893-917.

Gill S, Loprinzi CL, Sargent DJ, et al (2004). Pooled analysis of fluorouracil-based adjuvant therapy for stage II and III colon cancer: who benefits and by how much? J Clin Oncol, 22, 1797-806.

Gray R, Barnwell J, McConkey C, et al (2007) Adjuvant chemotherapy versus observation in patients with colorectal cancer: a randomised study. Lancet, 370, 2020-9.

Kuebler JP, Wieand HS, O'Connell MJ, et al (2007). Oxaliplatin combined with weekly bolus fluorouracil and leucovorin as surgical adjuvant chemotherapy for stage II and III colon cancer: results from NSABP C-07. J Clin Oncol, 25, 2198-204.
Labianca R, Nordlinger B, Beretta GD, et al (2013). Early colon cancer: ESMO Clinical Practice Guidelines for diagnosis, treatment and follow-up. Ann Oncol, 24, 64-72.

Lai JH, Zhou YJ, Bin D, Qiangchen, Wang SY (2014). Clinical significance of detecting lymphatic and blood vessel invasion in stage II colon cancer using markers D2-40 and CD34 in combination. Asia Pac J Cancer Prev, 15, 1363-70.

Meng J, Lu XB, Tang YX, et al (2013). Effects of allogeneic blood transfusion in patients with stage II colon cancer. Asia Pac J Cancer Prev, 14, 347-50.

Moertel CG, Fleming TR, Macdonald JS, et al (1990). Levamisole and fluorouracil for adjuvant therapy of resected colon carcinoma. $N$ Engl J Med, 322, 352-8.

O'Connor ES, Greenblatt DY, LoConte NK, et al (2011). Adjuvant chemotherapy for stage II colon cancer with poor prognostic features. J Clin Oncol, 29, 3381-8.

Ozdemir Y, Akin ML, Sucullu I, Balta AZ, Yucel E (2014). Pretreatment neutrophil/lymphocyte ratio as a prognostic aid in colorectal cancer. Asia Pac J Cancer Prev, 15, 2647-50.

Quah HM, Chou JF, Gonen M, et al (2008). Identification of patients with high-risk stage II colon cancer for adjuvant therapy. Dis Colon Rectum, 51, 503-7.

Ribic CM, Sargent DJ, Moore MJ, et al (2003). Tumor microsatellite-instability status as a predictor of benefit from fluorouracil-based adjuvant chemotherapy for colon cancer. N Engl J Med, 349, 247-57.

Sargent DJ, Marsoni S, Monges G, et al (2010). Defective mismatch repair as a predictive marker for lack of efficacy of fluorouracil-based adjuvant therapy in colon cancer. $J$ Clin Oncol, 28, 3219-26.

Siegel R, Desantis C, Jemal A,(2014). Colorectal cancer statistics, 2014. CA Cancer J Clin, 64, 104-17.

Talaiezadeh A, Tabesh H, Sattari A, Ebrahimi S (2013). Cancer incidence of southwest of Iran: first report from Khuzestan population-based cancer registry, 2002-2009. Asia Pac J Cancer Prev, 14, 7517-22.

Tournigand C, André T, Bonnetain F, et al (2012). Adjuvant therapy with fluorouracil and oxaliplatin in stage II and elderly patients (between ages 70 and 75 years) with colon cancer: subgroup analyses of the multicenter international study of oxaliplatin, fluorouracil, and leucovorin in the adjuvant treatment of colon cancer trial. J Clin Oncol, 30, 3353-60.

Trivedi H, Chamarthy U, Dicarlo L, Herman J, Srkalovic G (2014). Prognostic factors of overall survivall for patients with stage II colon cancer. Acta Med Acad, 43, 134-43.

Wilkinson NW, Yothers G, Lopa S, et al (2010). Long-term survival results of surgery alone versus surgery plus 5 fluorouracil and leucovorin for stage II and stage III colon cancer: pooled analysis of NSABP C-01 through C-05. A baseline from which to compare modernadjuvant trials. Ann Surg Oncol, 17, 959-66.

Wu X, Zhang J, He X, et al (2012). Postoperative adjuvant chemotherapy for stage II colorectal cancer: a systematic review of 12 randomized controlled trials. J Gastrointest Surg, 16, 646-55.

Yothers G, O'Connell MJ, Allegra CJ, et al (2011). Oxaliplatin as adjuvant therapy for colon cancer: updated results of NSABP C-07 trial, including survival and subset analyses. J Clin Oncol, 29, 3768-74. 